Sportwiss 2012 - 42:151-152

DOI 10.1007/s12662-012-0261-9

Online publiziert: 25. September 2012

(c) Springer-Verlag 2012

\author{
Ralf Brand ${ }^{1}$. Claudia Voelcker-Rehage ${ }^{2}$. Gertrud Pfister ${ }^{3} \cdot$ John Hoberman ${ }^{4}$ \\ 1 Department für Sport- und Gesundheitswissenschaften, Universität Potsdam, Potsdam \\ 2 Jacobs Center für Lebenslanges Lernen, Jacobs University Bremen, Bremen \\ ${ }^{3}$ Department of Exercise and Sport Sciences, University of Copenhagen, Copenhagen \\ ${ }^{4}$ Department of Germanic Studies, The University of Texas at Austin, Austin
}

\section{Geistes- und sozialwissenschaftliche Aspekte von Doping und Anti- Doping im Sport}

Psychology of Sport and Exercise haben zur Einreichung von Beiträgen für ein Schwerpunktheft zum „performance-enhancement by doping" aufgerufen; es soll im Frühjahr 2013 erscheinen.

Nun, das Themenheft der Sportwissenschaft halten Sie schon heute in ihren Händen. Es liegt weit vorne in diesem Trend, weil es die geistes- und sozialwissenschaftlichen Aspekte der Doping- und Anti-Dopingforschung fokussiert. Wir möchten behaupten, dass mit den hier versammelten Beiträgen sogar Perspektiven auf einige, bisher noch kaum beachtete Zusammenhänge eröffnet werden.

Die Resonanz auf den Aufruf zur Einreichung von Manuskripten bei der Sportwissenschaft war hinreichend groß. Insgesamt wurden 13 Beiträge eingereicht, zu denen 30 unabhängige Erstgutachten (im Blind-peer-review-Verfahren) eingeholt wurden. Fünf Beiträge wurden für dieses Themenheft ausgewählt. Einige mussten abgelehnt werden, einzelne befinden sich noch in Revisionsschleifen. Redaktionell wäre des Weiteren festzuhalten, dass es uns wiederum nicht gelungen ist, die eine oder andere Autorengruppe für einen englischsprachigen $\mathrm{Pu}$ blikationsversuch zu gewinnen. Dies hätte die Reichweite des Themenhefts und der darin vorgestellten Forschung zweifellos erhöht. Es bleibt uns die Zuversicht, dass sich die Dinge auch mit Blick auf eine verbesserte internationale Rezipierbarkeit bald in eine gute Richtung weiterentwickeln mögen.

Neben den vielfältigen (eher naturwissenschaftlichen) Forschungsaktivitäten zur Wirkung und dem Einsatz von Dopingmitteln, zu den gesundheitsschädlichen Auswirkungen oder den Möglichkeiten biochemischer Nachweise in Dopingproben, sollen im vorliegenden Heft nun also Forschungsergebnisse zu den gesellschaftlichen Ursachen von Doping, zu historischen Entwicklungen, zum Fitnessdoping und seinen Hintergründen sowie zum Umfeld von Doping und Anti-Doping zusammengeführt werden. Auslösenden Anschub gab das vom Deutschen Bundestag gewollte, vom DOSB begleitete und vom Bundesinstitut für Sportwissenschaft (BISp) geförderte mehrjährige Forschungsprojekt zur Aufarbeitung der Dopinggeschichte in Westdeutschland/BRD. Der weiterführenden Initiative des ehemaligen Herausgeberteams der Sportwissenschaft (um die geschäftsführenden $\mathrm{He}$ rausgeber Michael Krüger und Eike Emrich) ist es zu verdanken, dass dieser Anschub in entsprechende Arbeitsprozesse mündete.

Die Forschungsberichte von Marcel Reinold (gemeinsam mit Christian Becker und Stefan Nielsen) und Henk Erik Meier (gemeinsam mit Anica Rose und Stefanie Worboschil) gingen aus dem genannten Forschungsprojekt hervor, sie arbeiten die Entstehung und Entwicklung von Doping und Anti-Doping vor dem Hintergrund des historisch-politischen 
und gesellschaftlichen Kontextes der Bundesrepublik Deutschland seit den 1950er Jahren heraus und berücksichtigen dabei die Zusammenhänge zur historisch schon gründlicher aufgearbeiteten Dopingentwicklung in der DDR. Im Mittelpunkt steht zum einen das Verhältnis von Sport und Politik und zum anderen die öffentliche Wahrnehmung, Rezeption und Bewertung von Doping. Deutlich wird, dass Doping nicht nur ein reales, sondern v. a. ein sozial und gesellschaftlich konstruiertes Problem ist, für das die Medien Mitverantwortung tragen.

Des Weiteren beleuchtet David Müller, Manager für Prävention und Öffentlichkeit bei der österreichischen Nationalen Anti-Doping Agentur (NADA), einige der schier unlösbaren Probleme der AntiDopingpolitik. Beachtenswert ist sein Essay nicht zuletzt deshalb, weil er darin aus sehr unmittelbaren praktischen Erfahrungen aus seiner täglichen Arbeit schöpfen kann. Die sportsoziologische Arbeit von Mischa Kläber zeigt, dass EnhancementPraktiken nicht nur im Hochleistungssport beobachtet und erforscht werden sollten. Er untersucht die EnhancementBemühungen von Sporttreibenden in Fitnessstudios und problematisiert v. a. die Bereitschaft von Medizinerinnen und Medizinern, am Traum von der ewigen Fitness mitzuwirken. Schließlich stehen im Beitrag von Monika Frenger (gemeinsam mit Werner Pitsch und Eike Emrich) ökonomische Aspekte des Dopings im Mittelpunkt. Hier werden wirtschaftliche Anreize thematisiert und empirische Zusammenhänge aufgezeigt, die wahrscheinlich einen maßgeblichen Teil der Dopingaffinität von Sportlerinnen und Sportlern erklären.

Von Seiten des Herausgeberteams der Sportwissenschaft bleibt hinzuzufügen, dass unser besonderer Dank den beiden Gastherausgebern dieses Themenhefts, Gertrud Pfister (Kopenhagen) und John Hoberman (Austin, Texas) gilt. Es war uns Ehre und Auszeichnung, auf die Arbeit und uneingeschränkte Unterstützung zweier solchermaßen international renommierter Experten zu geistes- und sozialwissenschaftlichen Fragen im The- menbereich Doping und Anti-Doping im Sport bauen zu können! Mit Blick auf das zum Jahresende 2012 erscheinende Heft 4 unserer Zeitschrift freut es uns, schon ankündigen zu dürfen, dass sich Wolfgang Schöllhorn (Universität Mainz) bereiterklärt hat, ausführlich auf die von Stefan Künzell (Universität Augsburg) und Ernst-Joachim Hossner (Universität Bern) in Heft 2/2012 geäußerte Kritik zum bewegungswissenschaftlichen Ansatz des differenziellen Lernens und Lehrens im Sport einzugehen.

Gemeinsam mit unseren Gastherausgebern wünschen wir nun aber allen Leserinnen und Lesern unserer Zeitschrift eine gewinnbringende Lektüre der in diesem Themenheft „Geistes- und sozialwissenschaftliche Aspekte von Doping und Anti-Doping im Sport" versammelten Beiträge.

Wir freuen uns auf Ihr Feedback, Ihre

Ralf Brand und Claudia Voelcker-Rehage für das Herausgeberteam der Sportwissenschaft Gertrud Pfister und John Hoberman Gastherausgeber des Themenhefts

\section{Korrespondenzadresse}

Prof. Dr. Ralf Brand

Department für Sport-

und Gesundheitswissenschaften,

Universität Potsdam,

Am Neuen Palais 10, 14469 Potsdam

ralf.brand@uni-potsdam.de 